\title{
Alimentación del pez tropical Gobioides broussonnetii (Pisces: Gobiidae) en la Laguna de Unare, Venezuela
}

\author{
José Rodríguez ${ }^{1,2}$ \& Estrella Villamizar ${ }^{1}$ \\ 1 Departamento de Biología, Facultad de Ciencias y Tecnología, Apartado 2005, Universidad de Carabobo, Valencia, \\ Venezuela. Tel: (058241) 868-84-62; stegastes@hotmail.com; jgrodrigu@uc.edu.ve \\ 2 Laboratorio de Ecosistemas Marino Costeros (LEMAC), IZT, Facultad de Ciencias, Universidad Central de Venezuela, \\ Apartado 47058, Caracas 1041-A Venezuela. Fax: (058212) 605-12-04; evillami@strix.ciens.ucv.ve
}

Recibido 13-III-2002. Corregido 26-VIII-2005. Aceptado 09-VIII-2006.

\begin{abstract}
Diet of the tropical fish Gobioides broussonnetii (Pisces: Gobiidae) at Laguna de Unare, Venezuela. Unare Lagoon is one of the most important fishery coastal ecosystems in Venezuela. For the past 10 years, changes in its hydrological regime have affected the physiochemical characteristics of the water with important consequences on its faunal composition and abundance. An example of such variation is the abundant presence of a fish species, Gobioides broussonnetii, recently registered in this ecosystem. The ecology of this species must be studied to identify new ecological processes occurring in the lagoon. Detritus is the most important component of the G. broussonnetii diet, followed by plant and animal items. The species is a primary detritivorous consumer and this may be associated with its activity pattern (morning): It may be an occasional visitor because it was not captured regularly in the monthly samplings. Rev. Biol. Trop. 54 (4): 1093-1098. Epub 2006 Dec. 15.
\end{abstract}

Key words: Gobioides broussonnetii, detritivorous, coastal lagoon, diet, trophic status.

Las lagunas costeras son consideradas entre los ecosistemas de mayor productividad en el planeta y las mismas representan áreas de gran potencial pesquero. Sin embargo, esta productividad puede verse afectada producto de las actividades antrópicas que se desarrollan en las mismas lagunas o en sus adyacencias. Este es el caso de la Laguna de Unare, la cual se encuentra localizada en la costa centro-oriental de Venezuela, entre los

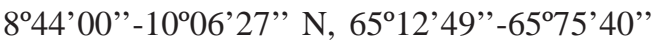
W. Presenta un área superficial de 4750 ha con $22 \mathrm{~km}$ de longitud y $6 \mathrm{~km}$ de ancho máximo (Roa 1989). Esta laguna se separa del océano mediante una barra arenosa de 300 a $600 \mathrm{~m}$ de ancho, y presenta dos bocas de comunicación con el mar, las cuales se encuentran en la actualidad completamente cerradas, producto de una carretera que se construyó sobre la misma. Esta condición y un continuo aporte de agua dulce desde el río Unare producto de su represamiento, ha generado cambios en este sistema estuarino, siendo el más notorio de todos la disminución de la salinidad (Roa 1993). Esto ha generado variaciones en la composición y abundancia relativa de las especies, siendo un ejemplo de esto la aparición del pez Gobioides broussonnetii (Lacepéde 1800), una especie perteneciente a la familia Gobiidae, cuyo género se caracteriza por tener una sola aleta dorsal larga y continua (Cervigón 1994) y que dentro de la familia es la especie que suele alcanzar las mayores tallas.

Esta especie no fue informada por Mago (1965) en su contribución a la sistemática de los peces de la Laguna de Unare, lo que hace pensar que es de aparición reciente. La presencia y reciente incremento en la abundancia 
de G. broussonnetii puede ser atribuido a los cambios en los parámetros fisicoquímicos mencionados anteriormente. Por esta razón es importante estudiar la ecología de esta especie, a fin de obtener datos que contribuyan al conocimiento de nuevos procesos ecológicos que pudiesen estar ocurriendo en este sistema.

El objetivo principal de este trabajo es estudiar los hábitos alimentarios de G. broussonnetii, con el fin de determinar su posición trófica en la laguna así como el papel ecológico que esta especie pueda estar desempeñando en este sistema.

\section{MATERIALES Y MÉTODOS}

Con el fin de evaluar la dieta de G. broussonnetii, se analizaron 93 individuos recolectados mensualmente en horas del amanecer, desde agosto de 1993 hasta marzo de 1994 en la boca occidental de la Laguna de Unare, en una zona conocida como la Cerca. Los individuos fueron capturados con una atarraya de apertura de malla de $3.5 \mathrm{~cm}$ y preservados con formalina al $10 \%$ para su traslado al laboratorio. El horario de captura se seleccionó considerando las observaciones de dos seguimientos previos de las pesquerías en la zona por un periodo de $24 \mathrm{~h}$.

Se analizaron los contenidos estomacales utilizando el índice de Dominancia numérica (DN), también conocido como Frecuencia numérica (Fn) y la Frecuencia de aparición estomacal (Fa) (Hyslop 1980). Para el cálculo de estos índices, se requiere contabilizar el número de individuos de cada uno de los ítems alimenticios. El número de células del componente fitoplanctónico se estimó con la metodología propuesta por Wetzel y Linkens (1991) con cámaras de sedimentación y microscopio invertido. Esta misma metodología se utilizó para determinar el número de organismos del componente zooplanctónico, donde se cuenta el total de organismos contenidos en el volumen de una cámara de Sedwick-Rafter. Tanto para el fitoplancton como el zooplancton se analizaron tres alícuotas por estómago.
Para establecer la importancia de cada ítem alimenticio en la dieta de una especie, es aconsejable, además de la frecuencia numérica y la frecuencia de aparición, estimar un tercer parámetro que mida la importancia de los mismos, e integrar los tres parámetros en un índice de importancia relativa. Por lo general suele incluirse como tercer parámetro el porcentaje volumétrico o gravimétrico de cada ítem. El problema en nuestro caso radica en que el componente planctónico está representado por individuos de muy pequeño tamaño y escasas cantidades, lo que dificulta separarlo por especies y hacer determinaciones de volumen o peso. Adicionalmente, uno de los componentes más importantes en la dieta de esta especie es el detritus, tal como lo informan Acero y Granados (1989-1900), el cual no se puede contar. Por ambas razones se estimó la importancia relativa de los ítems en la dieta de esta especie utilizando el método de superficie de “punto intercepto” (POSU) de Weinberg (1981) utilizado para estudios en comunidades marinas, pero modificado para el análisis de estos contenidos estomacales. Así, se utilizó una cámara de Sedwick-Rafter cuadriculada, con un total de 48 interceptos o puntos de muestreo. Para cada contenido estomacal se tomó una alícuota de $0.2 \mathrm{ml}$ la cual se homogeneizó con agua destilada al volumen de la cámara (1 ml) y una vez colocada la alícuota en la cámara se determinó la frecuencia de cada ítem (detritus, zooplancton y fitoplancton) bajo el microscopio, contando el número de interceptos ocupados por un determinado ítem con relación al total de puntos. Con estos datos se calculó una frecuencia absoluta y luego la frecuencia relativa de todos los ítems.

Con los datos de DN, Fa estomacal y Frecuencia relativa de los diferentes ítems, se procedió a calcular el índice de importancia relativa de Windell, IRI (Hyslop 1980), realizando una modificación, siendo los datos volumétricos o gravimétricos sustituidos por los valores obtenidos con el método de Frecuencia de superficie de punto intercepto. Se calculó un primer $\mathrm{IRI}_{1}$ para cada uno de los ítems, sin tomar en cuenta la frecuencia numérica, tal 
como lo hizo Ponte (1990), ya que este parámetro no es posible estimarlo para el componente detritus. Este primer índice nos permite establecer la importancia del detritus dentro de la dieta de esta especie.

Índice original Índice modificado

$$
\mathrm{RI}_{\mathrm{a}}=\left(\% \text { volumétrico } \mathrm{a}+\mathrm{Fn}_{\mathrm{a}}\right) * \mathrm{Fa}_{\mathrm{a}}
$$

$$
\begin{gathered}
\mathrm{RI}_{1 \mathrm{a}}=\left(\text { Frecuencia Punto Intercepto }{ }_{\mathrm{a}}\right) * \mathrm{Fa}_{\mathrm{a}} \\
\mathrm{IRI}_{1 \mathrm{a}}=\frac{\mathrm{RI}_{1} \text { del ítem a }}{\mathrm{RI}_{1}}
\end{gathered}
$$

Para el componente planctónico, si bien se puede establecer la importancia de cada ítem con este primer índice (IRI $)_{1}$ ), se calculó un segundo $\mathrm{IRI}_{2}$, donde si se toman en cuenta los valores de Fn, lo que hace más confiable el cálculo, al menos para estos ítems.

$$
\begin{gathered}
\mathrm{RI}_{2 \mathrm{a}}=\left(\text { Frecuencia Punto Intersepto } \mathrm{a}+\mathrm{Fn}_{\mathrm{a}}\right) * \mathrm{Fa}_{\mathrm{a}} \\
\mathrm{IRI}_{2 \mathrm{a}}=\frac{\mathrm{RI}_{2} \text { del ítem a }}{\mathrm{RI}_{2}}
\end{gathered}
$$

Combinando ambos índices, se procedió a determinar la importancia de cada uno de los ítems alimenticios en la dieta de G. broussonnetii. El IRI ${ }_{1}$ permite establecer la importancia del detritus con respecto a los demás ítems y con el $\mathrm{IRI}_{2}$ se ordena la importancia de los diferentes ítems que conforman el componente planctónico.

Para establecer si existen variaciones mensuales en la dieta de esta especie, se utilizaron los datos de frecuencia de punto intercepto (POSU) y se aplicó un análisis de tablas de contingencia (Grillet y Barrera 1997).

El horario de mayor actividad alimentaria de esta especie se cuantificó con el peso seco de cada contenido estomacal, valor con el que se calculó el índice de cantidad de alimento (Ica) (Hyslop 1980), verificando la significancia de los mismos con el análisis de Kruskal-Wallis.

Para establecer el horario de mayor actividad de esta especie se graficó el número de ejemplares capturados en los diferentes horarios ( $\mathrm{N}^{\mathrm{o}}$ peces/horario), y se realizó una correlación con el índice de cantidad de alimento para verificar si la mayor actividad está relacionada con su proceso alimentario.

\section{RESULTADOS}

Los resultados del Índice de Windell, tanto el IRI ${ }_{1}$ como el IRI ${ }_{2}$, obtenidos en el análisis de la dieta de $G$. broussonnetii permiten agrupar las presas consumidas en ocho categorías o ítems alimenticios (Cuadro 1).

Es importante señalar que se incluyó en los análisis un noveno componente, denominado componente inorgánico, representado principalmente por arena, el cual aunque no representa un recurso energético para el pez, es importante en el proceso digestivo de muchas especies por su acción fragmentadora, lo cual facilita el proceso digestivo en peces detritívoros y planctívoros.

Los resultados del Î́ndice de Windell ( IRI $_{1}$ ) (Fig. 1) indican que el detritus, con un valor promedio de $69.38 \%$ es el componente más importante en la dieta de esta especie, seguido

\section{CUADRO 1}

Ítems alimenticios en la dieta de G. broussonnetii

TABLE 1

Food items in the diet of $\mathrm{G}$. broussonnetii

Ítem alimenticio

Componentes

\section{Detritos}

Cianofitas

Anabaena sp. Chroochocus sp.

Nitzchia sp.

Diatomeas Navicula sp. Amphiprora alata

Clorofitas Mougeotia sp. Odeogonium sp.

\section{Huevos}

Rotíferos

Brachionus plicatilis

Keratella sp.

Copépodos

Oitona sp.

Otros
Cladóceros

Larvas de dípteros 


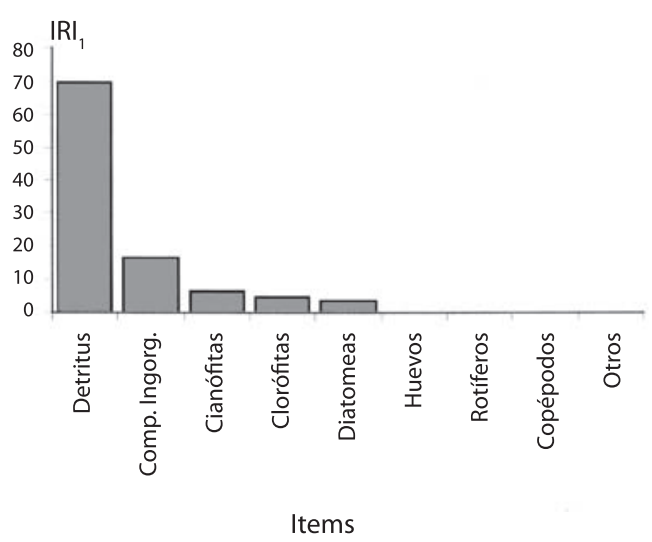

Fig. 1. IRI $I_{1}$ promedio de todos los ítems alimenticios encontrados durante todo el muestreo.

Fig. 1. Average $\mathrm{IRI}_{1}$ of food items found during the sampling period.

luego por el componente fitoplanctónico (cianofitas, clorofilas y diatomeas $3.31 \%$ ) y por último el componente zooplanctónico, con valores bastante bajos con relación a los otros ítems (huevos, rotíferos y copépodos). Los resultados del $\mathrm{IRI}_{2}$ (Fig. 2), reordenan la importancia de los ítemes contables dando como resultado que las diatomeas con un valor de $\mathrm{IRI}_{2}$ de $31.32 \%$ son más importantes que las clorofitas con un valor de $28.74 \%$, mientras que las cianofitas se mantienen como las más importantes con un $39.24 \%$ de $\mathrm{IRI}_{2}$. Para el componente zooplanctónico la importancia relativa no cambia.

Tomando en cuenta los resultados de ambos índices, se puede establecer el siguiente orden de importancia para los ítems consumidos por G. broussonnetii:

Detritus $>$ Cianofitas $>$ Diatomeas $>$ Clorofitas $>$ Huevos $>$ Rotíferos $>$ Copépodos.

Con relación al componente inorgánico (arena), su incorporación es bastante alta, con un promedio de $16.44 \%$, siendo solo superado por el valor del detritus (Fig. 1).

Los resultados del análisis mensual de la dieta revelan perfiles de consumo significativamente diferentes $\left(X^{2}=5724.93\right.$, $\mathrm{df}=24, \mathrm{p}<0.05)$, existiendo diferencias tem-

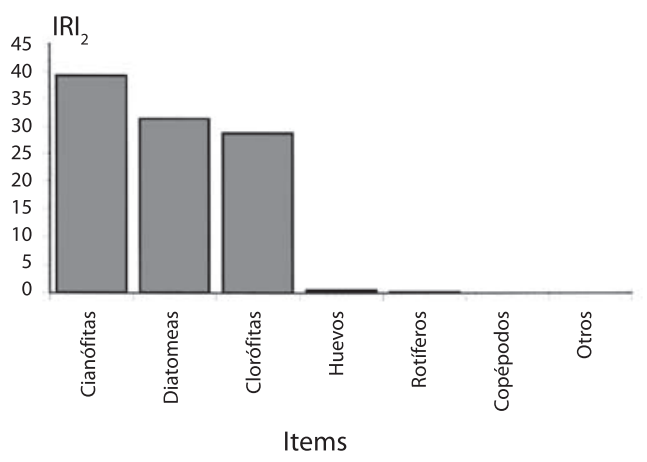

Fig. 2. $\mathrm{IRI}_{2}$ promedio de ítems alimenticios cuantificables encontrados durante todo el muestreo.

Fig. 2. Average $\mathrm{IRI}_{2}$ of quantifiable food items found during sampling period.

porales en el consumo de los diferentes ítems alimenticios, donde la mayor incorporación de detritus se da durante el mes de noviembre (Fig. 3). Igualmente se da una mayor ingesta de cianofitas en septiembre y de clorofilas para el mes de marzo (Fig. 3).

Los resultados del índice de cantidad de alimento (Ica) (Fig. 4) revelan la presencia de un máximo de alimentación entre las 5:00 y 8:00 am, donde el mayor valor se alcanza entre las 6:00 y 7:00 am. Estos valores son significativamente superiores a los obtenidos en los otros horarios (Kruskal-Wallis, $\mathrm{H}=19.71$; $\mathrm{p}=0.0198$ ), por lo cual podemos decir que estas son las horas de máxima alimentación de G. broussonnetii.

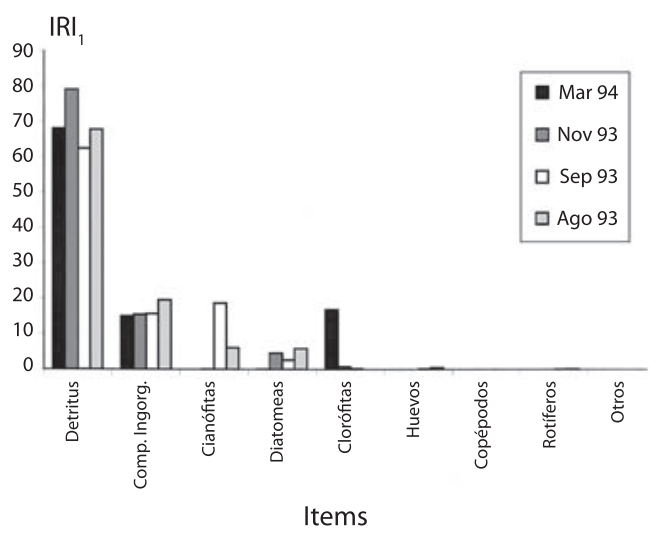

Fig. 3. Variación mensual en la dieta.

Fig. 3. Monthly diet variation. 


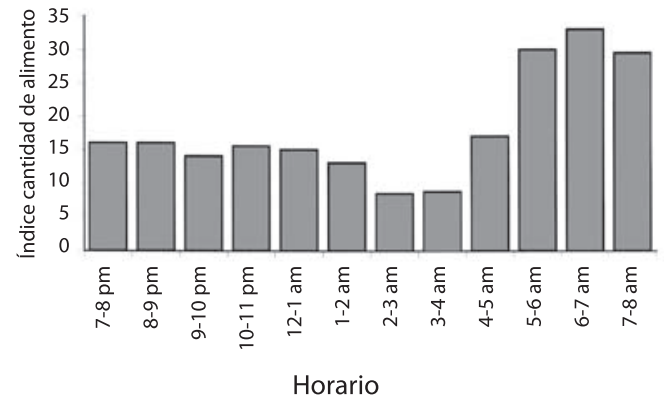

Fig. 4. Horario de alimentación de G. broussonnetii.

Fig. 4. Feeding schedule of G. broussonnetii.

Un resultado similar se obtiene con el horario de actividad de esta especie, donde las mayores capturas se dan entre las 5:00 y 8:00 am. (Fig. 5). La correlación entre el horario de actividad de la especie y el horario de alimentación fue significativa (Spearman, $\mathrm{r}=0.92$ ). Entre las 8:00 am. y 7:00 pm. no se capturaron ejemplares en ningún muestreo, a pesar de que estos fueron realizados durante 24 h. Igualmente a pesar de haberse realizado muestreos mensuales la especie no fue capturada durante todos los meses.

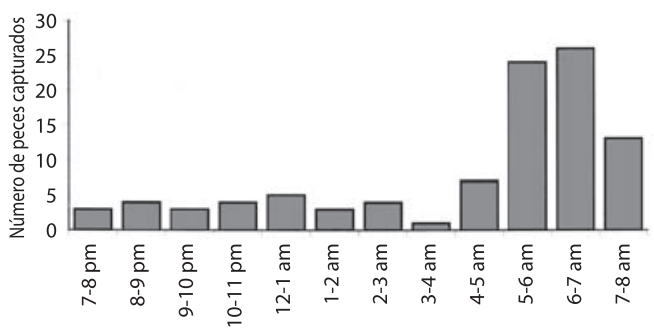

Horario

Fig. 5. Horario de actividad de G. broussonnetii.

Fig. 5. Activity schedule of G. broussonnetii.

\section{DISCUSIÓN}

Estos resultados indican que G. broussonnetii es un consumidor primario del tipo detritívoro, según la clasificación de Yáñez-Arancibia (1978), que incorpora junto al detritus un componente vegetal y animal complementario, probablemente asociado a este detritus. Así, la presencia de microalgas y del componente animal en la dieta de esta especie, parece estar determinada más que por selectividad, a la asociación de dichos componentes al detritus. Estos resultados concuerdan con los obtenidos por Acero y Granados (1989-1990), quienes concluyeron que $G$. broussonnetii es un consumidor primario detritívoro, aunque las proporciones e ítems complementarios son diferentes a los encontrados en este trabajo.

Esta especie cumple un papel ecológico relevante, transformando el potencial energético del detritus por consumo directo, permitiendo así la progresión de los nutrientes en la trama trófica hacia niveles superiores, tal como lo señalan Yáñez-Arancibia y Nugent (1977). Dicha importancia se incrementa al tomar en cuenta lo abundante que es el detritus en este ecosistema costero, dado el gran aporte de material alóctono proveniente del río Unare, el cual representa en la actualidad el cuerpo de agua con mayor influencia sobre la laguna, ya que las bocas de comunicación con el mar están cerradas.

Esta especie manifiesta variaciones mensuales en la dieta, sin embargo estas variaciones están principalmente referidas a las proporciones de consumo de los diferentes ítems alimenticios, lo cual pudiese estar directamente relacionado a la disponibilidad de los mismos en el ambiente, ya que es un sistema estuarino donde el aporte de material aloctono proveniente del río varía con la época del año, dependiendo de la intensidad de las precipitaciones a lo largo de toda la cuenca del río Unare. Así la mayor ingesta de detritus hacia el mes de noviembre puede estar asociada a los máximos de precipitación que también se dan en estos meses en las cabeceras del río Unare, lo que puede generar un mayor aporte de este material a la laguna.

Finalmente, G. broussonnetii se puede caracterizar como una especie de hábitos matutinos, ya que su mayor actividad se presenta en las primeras horas de la mañana y la misma está relacionada a su proceso alimentario tal como 
lo revelan los resultados. El resto del día no se pudo determinar la ubicación de esta especie, ya que la misma no fue capturada y no sabemos si permanece en la laguna, remonta el río o vuelve al mar. Por otro lado, la alta turbidez del sistema impide hacer seguimientos visuales de la misma. Adicionalmente esta especie no fue capturada durante todos los meses de muestreo, por lo cual puede catalogarse como un visitante ocasional de la laguna más que un residente estuarino; sin embargo, este resultado puede ser el reflejo del arte de pesca empleado y quizás se requiera de un mayor esfuerzo de muestreo para llegar a una conclusión más acertada.

\section{AGRADECIMIENTOS}

A Carmen Teresa Rodríguez por su valiosa colaboración en la captura de los ejemplares durante todos los muestreos. A Pedro Roa, in memoriam, por habernos dado a conocer la importancia de los sistemas lagunares costeros de Venezuela.

\section{RESUMEN}

La Laguna de Unare es uno de los ecosistemas costeros más importantes de Venezuela desde el punto de vista pesquero. Durante las décadas de 1980 y 1990, los cambios en su régimen hidrológico han afectado las características fisicoquímicas del agua, con consecuencias sobre la composición y abundancia de la fauna asociada. Un ejemplo de estas variaciones es la abundante presencia de Gobioides broussonnetii, una especie de pez registrada recientemente en este sistema. Por tal razón es importante identificar nuevos procesos ecológicos que pudiesen estarse dando en la laguna. El detrito es el componente más importante en la dieta de $G$. broussonnetii, seguido por un componente vegetal y animal asociado a este detrito: esta especie es un consumidor primario detritívoro, lo que podría relacionarse con su horario matutino de actividad. Esta especie no es capturada regularmente en los muestreos mensuales, lo cual hacer pensar que es un visitante ocasional de la laguna.

Palabras clave: Gobioides broussonnetii, detritívoro, laguna costera, alimentación, estado trófico.

\section{REFERENCIAS}

Acero, A. \& P. Granados. 1989-1990. Gobioides broussoneti Lacepede (Pisces: Gobiidae) en el Caribe Colombiano. An. Inst. Invest. Mar. Punta Betin. 1920: 197-200.

Cervigón, F. 1994. Los Peces Marinos de Venezuela. Tomo III. Ex-Libris, Caracas, Venezuela. 295 p.

Grillet, M. \& R. Barrera. 1997. Spatial and temporal abundance, substrate partitioning and species co-ocurrence in a guild of Neotropical blackflies (Diptera: Simuliidae). Hidrobiol. 345: 197-208.

Hyslop, E. 1980. Stomach contents analysis-a review of methods and their application. J. Fish Biol. 17: 411-429.

Lacepéde, B.G.E. 1800. Histoire Naturelle des poissons.Museé National d'Histoire Naturelle, Paris, Francia. 632 p.

Mago, F. 1965. Contribución a la sistemática y ecología de los peces de la Laguna de Unare, Venezuela. Bull. Mar. Sci. 15: 274-330.

Ponte, V. 1990. Recurso trófico utilizado por peces juveniles en dos áreas del delta inferior del Río Orinoco. Tesis de Licenciatura, Univ. Central de Venezuela, Venezuela. 89 p.

Roa, P. 1989. Resumen de las características generales de las lagunas costeras de Tacarigua, Unare y Piritu. In Proyecto “Aquila” GCP/RLA/075/ITA. Convenio FAO-Univ. Simón Bolívar. Caracas, Venezuela. 17 p.

Roa, P. 1993. La Transgresión Flamenca y la evolución de la Laguna de Unare, Venezuela. Bol. Inst. Oceanogr. Venez. Universidad de Oriente, Venezuela 29: 11-25.

Weinberg, S. 1981. A comparison of coral reef survey methods. Bijdr. Dierk. 51: 199-218.

Wetzel, R. \& G.E. Likens. 1991. Limnological analyses. Springer, Nueva York, EEUU. 391 p.

Yáñez-Arancibia, A. 1978. Taxonomía, ecología y estructura de las comunidades de peces en lagunas costeras con bocas efímeras del Pacifico de México. Centro Cien. del Mar y Limnol. Univ. Nal. Autón. México. 2303 p.

Yáñez-Arancibia, A. \& R. Nugent. 1977. El papel ecológico de los peces en estuarios y lagunas costeras. An. Centro Cien. del Mar y Limnol. Univ. Nal. Autón. México 4: 107-114. 